\title{
Crossing Disciplinary Boundaries to Understand Human Drivers of Environmental Threats*
}

\author{
Eugene A. Rosa \\ Department of Sociology \\ Washington State University \\ Pullman, WA 99164-4020 \\ rosa@wsu.edu
}

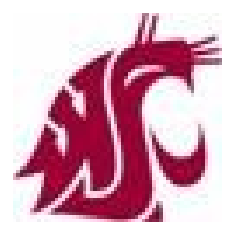

Thomas Dietz

Sociology and Environmental Science and Policy

Michigan State University

East Lansing, MI 48824

MICHIGAN STATE

Tdietzvt@gmail.com

*Paper presented at the annual meetings of the Ecological Society of America, Pittsburgh,

PA (1-6 August, 2010) 


\section{IF I HAD A HAMMER!}

“GIVE A LITTLE BOY A HAMMER, AND HE WILL FIND THAT

EVERYTHING HE ENCOUNTERS NEEDS POUNDING."

THE LAW OF INSTRUMENT, ABRAHAM KAPLAN (1964:28)*

QUESTION: IF EVERYTHING IS TACKS, DOES ONE EVER THINK OF A HAMMER?

*A VERSION IS ALSO ATTRIBUTED TO MARK TWAIN: "TO A MAN WITH A HAMMER EVERYTHING LOOKS LIKE A NAIL." 
OBSERVATION:

THE HUMAN DIMENSIONS OF GLOBAL ENVIRONMENTAL

CHANGE RESEARCH HAS EMPHASIZED THE TACKS (IMPACTS OF HUMAN DRIVERS) RATHER THAN THE HAMMER ITSELF (DRIVERS -ANTHROPOGENIC SOURCES—OF IMPACTS):

- LAND USE, LAND COVER CHANGE -ECOLOGICAL RISK: VULNERABILITY, RESILIENCY,

ADAPTATION

-INSTITUTIONAL ISSUES: PRACTICES

(1) COMMON POOL/PROPERTY

(2) ACTIONS TO ADDRESS IMPACTS 


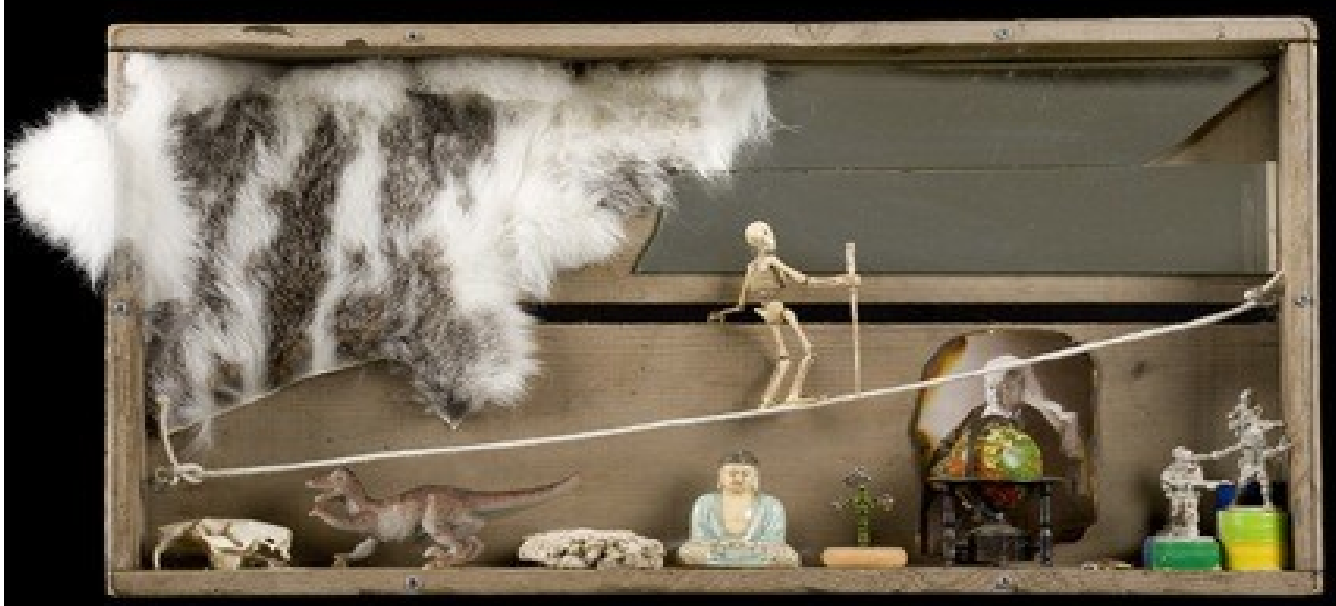

\section{Human Footprints on} the Global Environment

Threats to Sustainability

edited by Eugene A. Rosa, Andreas Diekmann, Thomas Dietz, and Carlo C. Jaeger 
Definition: Global Environmental Change

1. CUMULATIVE EFFECTS - EFFECTS THAT ARE

LOCAL IN DOMAIN BUT SO WIDELY REPLICATED THAT IN SUM THEY HAVE

GLOBAL

CONSEQUENCES.

EXAMPLES: TROPICAL DEFORESTATION, DESERTIFICATION, SPECIES LOSS,

DAMAGED

LOCAL ECOSYSTEMS, AND RESOURCE EXHAUTION.

2. SYSTEMIC EFFECTS - EFFECTS THAT OCCUR ON LARGE SPATIAL SCALES OR 
CHANS - COUPLED HUMAN AND NATURAL* SYSTEMS

THESE ARE INTEGRATED SYSTEMS IN WHICH PEOPLE INTERACT WITH NATURAL COMPONENTS

A. THEIR COMPLEXITY IS NOT WELL UNDERSTOOD

B. DUE TO TRADITIONAL SEPARATION OF SOCIAL AND ECOLOGICAL SCIENCES

C. MUCH GREATER UNDERSTANDING OF H->N THAN N->H

*OR ENVIRONMENTAL SYSTEMS

REFERENCES:

LIU, JIANGUO (JACK) ET AL.. 2007. "COMPLEXITY OF COUPLED HUMAN AND NATURAL SYSTEMS." SCIENCE 317: 1513-1516.

LIU, JIANGUO (JACK) ET AL.. 2007. "COUPLED HUMAN AND NATURAL SYSTEMS." AMBIO 36: 639-649. 
THE HAMMER: STIRPAT RESEARCH PROGRAM:

\author{
THOMAS DIETZ - MICHIGAN STATE \\ UNIVERSITY
}

\author{
EUGENE A. ROSA - WASHINGTON STATE \\ UNIVERSITY
}

RICHARD YORK - UNIVERSITY OF OREGON

무붐

KYLE KNIGHT - WASHINGTON STATE UNIVERSITY 


\section{ECOLOGICAL FIRST PRINCIPLES:}

NATURE PROVIDES ECOLOGICAL CAPITAL AND SERVICES.

FACTORS IMPACTING THEM ARE:

FOR ALL SPECIES: *(1) NUMBER OF MEMBERS

(2) GEOGRAPHIC SPACE

*(3) RATE RESOURCES ARE CONSUMED

FOR HIGHER ORDER *(4) TECHNOLOGY

SPECIES (HUMANS) (5) AMOUNT OF WASTE

*WHAT NORMAN MYERS CALLS THE "REDOUBTABLE TRIAD" 


$$
\mathbf{I}=\mathbf{P A T}
$$

Accounting Version

Impacts (I)

Population (P)

Affluence (A) or Consumption Technology (T) 
Typical Application:

Basic Identity:

$$
\mathbf{I}=\text { PAT }
$$

Form:

$T=I /\left(P^{*} A\right)$ SOLVE FOR $T$

Data:

Typically \% Changes

Example (Commoner 1992)

From 1950 to 1967 , following changes:

Production/

Synethic Pesticide Use Population Affluence Technology $+266 \%$ $+30 \%$ $+5 \%$ ?

$\mathrm{T}=3.66 /(1.3 \times 1.05)=2.68$

$T=2.68-1.00(100)=168 \%$ increase 
产 THE COMPASS

IPAT: ACCOUNTING VERSION (OR IDENTITY)

IMPACTS TO THE ENVIRONMENT $=f(\mathrm{P}, \mathrm{A}, \mathrm{T})$

THE GPS

STIRPAT: STOCHASTIC VERSION (ACCOUNTING FOR ERROR)

IMPACTS TO THE ENVIRONMENT $=f(\mathbf{P}, \mathbf{A}, \mathbf{T})+\varepsilon$ 


\section{STIRPAT ESTIMATION FORMULA:}

(a) $\operatorname{LOG} I=\operatorname{LOG} a+b \log P+c \log A+\log e$

\section{OR}

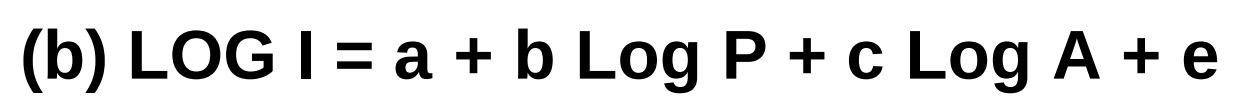

$$
\text { Where } \begin{aligned}
a & =\text { LOG } a \\
& e=\text { LOG } e
\end{aligned}
$$




\section{STIRPAT RESEARCH PROGRAM (NATION}

\section{STATES)}

STRESSORS EXAMINED (PUBLISHED):

$\mathrm{CO}^{2}$ (CARBON DIOXIDE)

$\mathrm{CH}^{4}$ (METHANE)

$\mathrm{NO}_{\mathrm{x}}$ (NITROGEN OXIDES)

$\mathrm{SO}^{2}$ (SULFUR DIOXIDE)

ODS (OZONE DEPLETING SUBSTANCES)

ECOLOGICAL FOOTPRINT (FT) - (TOTAL)

ECOLOGICAL FOOTPRINT (FT) - (PARTS)

ADDITIONAL STRESSORS EXAMINED (NOT YET PUBLISHED):

CARBON MONOXIDE

NON-METHANE VOLATILE ORGANIC COMPOUNDS 


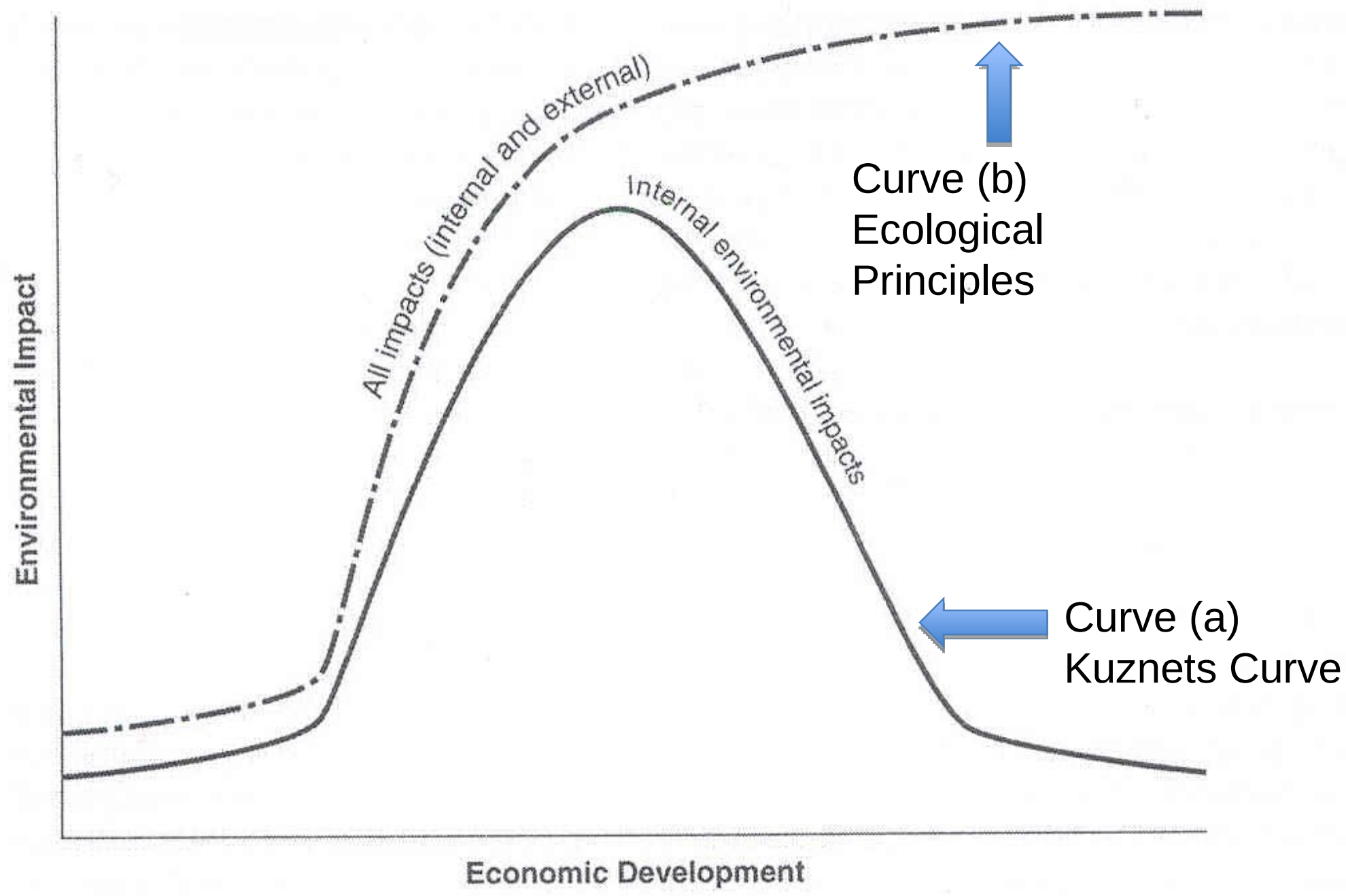

Figure 1. The Theoretical Effect of Economic Development on Envirommental Impact 


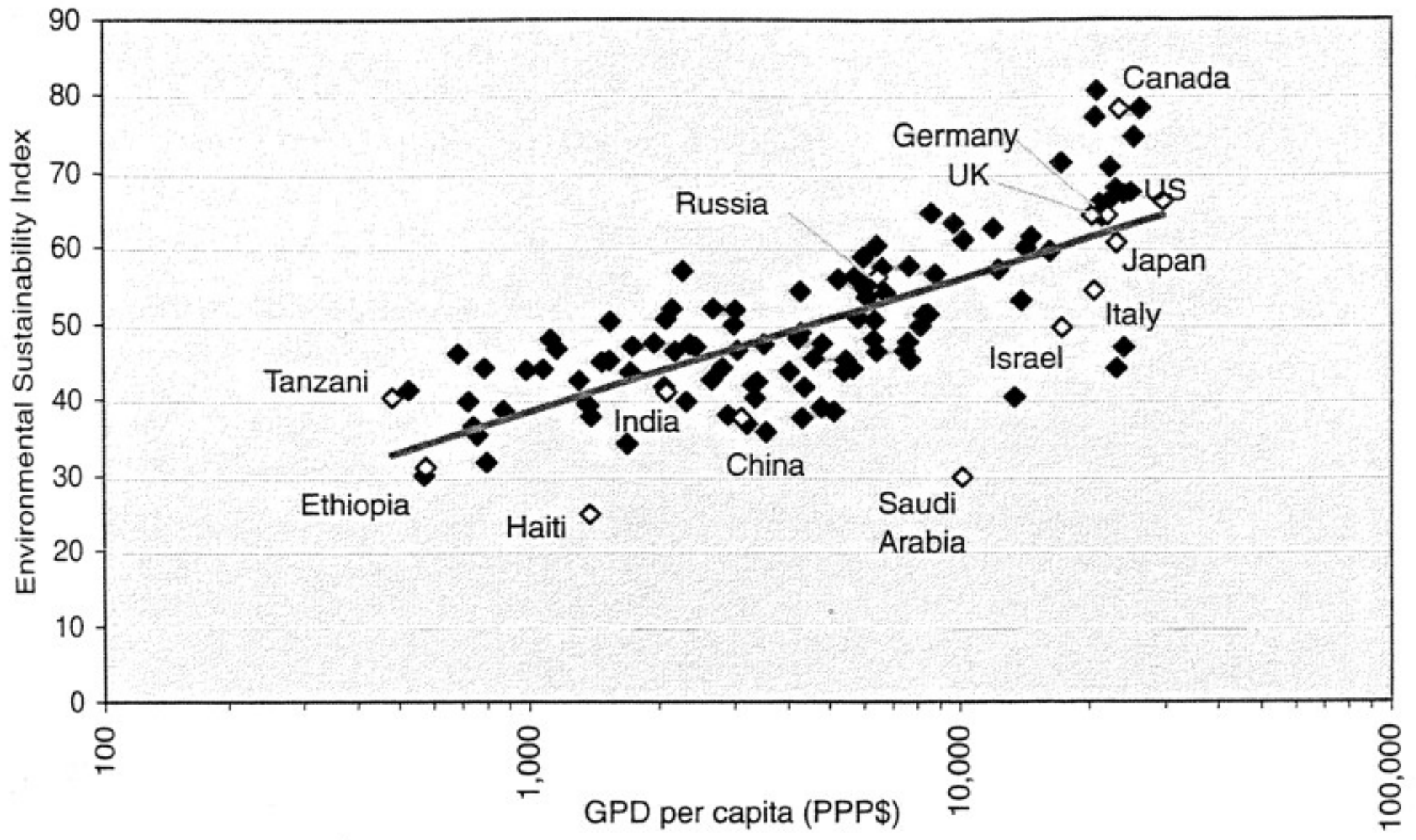

Figure 9 The connection for 117 nations between GDP per capita (current 1998 PPP\$) and the 2001 Environmental Sustainability Index, measuring 22 environmental dimensions on 67 variables. ${ }^{254} \mathrm{~A}$ best-fit line is displayed and various nations have been marked out. Source: WEF $2001 \mathrm{a} \&$ b, World Bank 2000c. 
ECOLOGICAL FOOTPRINT:

BAEIX IAEA: THE XYMYAATI

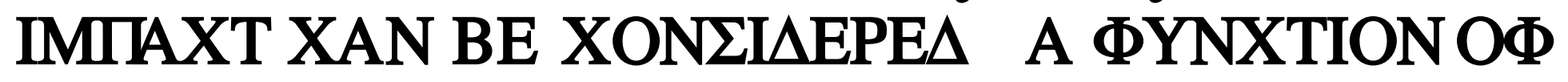

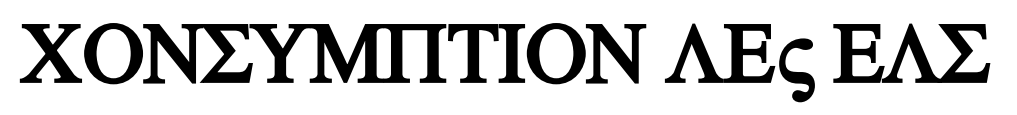

A XONGEPLION OФ XONLYMITTION INTO A

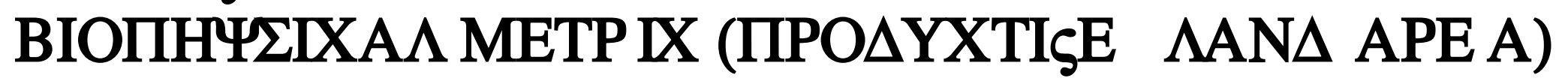

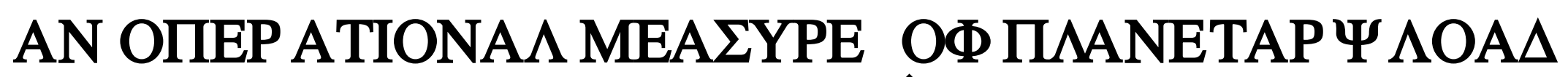

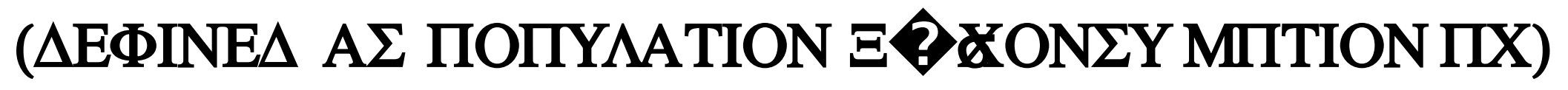

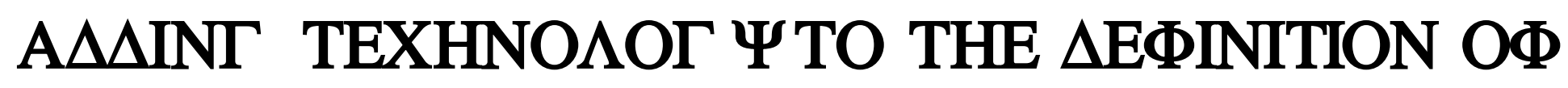
П $\Lambda$ ANETAP $\Psi \Lambda \mathrm{OA} \Delta$ PE$\Sigma Y \Lambda T \Sigma$ IN THE I=ПAT 


\section{THE ECOLOGICAL FOOTPRINT (EF):}

THE ECOLOGICAL FOOTPRINT (EF) IN ANY GIVEN YEAR IS THE AMOUNT

OF

BIOLOGICALLY PRODUCTIVE LAND (NATURE'S CAPITAL) NEEDED TO

REGENERATE

HUMAN CONSUMPTION, LIVING SPACE, AND TO ABSORB WASTES.

IMPORTANT CAVEAT:

THE EF MEASURES STRESS, PRESSURE OR LOADS ON NATURAL CAPITAL AND SERVICES, 
ECOLOGICAL FOOTPRINT (EF): ELEMENTS OF CONUMPTION* FIVE COMPONENTS

1. FOOD

2. HOUSING

3. TRANSPORTATION

4. CONSUMER GOODS

5. SERVICES

INCLUDING AN ACCOUNTING FOR WASTES 


\section{ECOLOGICAL FOOTPRINT (EF):BIOPHYSICAL UNITS}

\section{SIX COMPONENTS:}

1. ARABLE LAND (GROWING CROPS)

2. PASTURE LAND (ANIMAL GRAZING)

3. FORESTED LAND (TIMBER PRODUCTS)

4. SEA SPACE (PRODUCTIVE FISHING GROUNDS)

5. BUILT-UP LAND (INFRASTRUCTURE FOR

HOUSING, TRANSPORTATION, INDUSTRY, HYDROELECTRIC POWER)

6. ENERGY OR CARBON LAND (TO SEQUESTER $\mathrm{CO}_{2}$ EMISSIONS)

AGGREGATE EF: THE SEPARATE MEASURES CAN BE SUMMED INTO FN OVERAL ECOLOGICAL FOOTPRINT 


\section{Table 1. STIRPAT RESEARCH PROGRAM: EMPIRICAL STUDIES}

\begin{tabular}{|c|c|c|c|c|c|c|}
\hline $\begin{array}{l}\text { Publication } \\
\text { Date }\end{array}$ & Publication Outlet & Dependent Variables & $\begin{array}{l}\text { Number of } \\
\text { Nations }\end{array}$ & Data Year & $\begin{array}{l}\text { Kuznets } \\
\text { Curve }\end{array}$ & Noteworthy Features \\
\hline 1997 & PNAS $^{1}$ & $\mathrm{CO}_{2}$ Emissions & 111 & 1989 & $>\$ 10 \mathrm{~K}$ & $\begin{array}{l}\text { GDP/PC }<\$ 5 \mathrm{~K} \text { for } 75 \% \text { of } \\
\text { Nations }\end{array}$ \\
\hline 2003 & $\begin{array}{c}\text { International Journal } \\
\text { of Sociology \& social } \\
\text { Policy }\end{array}$ & $\begin{array}{l}\text { 1. } \mathrm{CO}_{2} \\
\text { 2. } \mathrm{CH}_{4} \\
\text { 3. } \mathrm{GWP}^{\star} \\
\end{array}$ & $\begin{array}{ll}\text { 1. } & 137 \\
\text { 2. } & 137 \\
\text { 3. } & 137\end{array}$ & $\begin{array}{ll}\text { 1. } & 1991 \\
\text { 2. } & 1991 \\
\text { 3. } & 1991\end{array}$ & $\begin{array}{c}\text { n.s. } \\
\text { n.s. } \\
+ \text { Quadratic }\end{array}$ & \\
\hline 2003 & $\begin{array}{c}\text { American Sociological } \\
\text { Review }\end{array}$ & $\begin{array}{l}\text { Total Ecological } \\
\text { Footprint (EF) }\end{array}$ & 142 & circa 1996 & + Quadratic & $\begin{array}{l}\text { No support for neo-liberal } \\
\text { modernization theories }\end{array}$ \\
\hline 2003 & Ecological Economics & $\begin{array}{l}\text { 1. } \mathrm{CO}_{2} \text { Emissions } \\
\text { 2. Energy } \\
\text { Footprint }\end{array}$ & $\begin{array}{l}\text { 1. } 146 \\
\text { 2. } 138\end{array}$ & $\begin{array}{l}\text { 1. } 1996 \\
\text { 2. } 1999\end{array}$ & $\begin{aligned} & >\$ 61 \mathrm{~K}^{1} \\
+ & \text { Quadratic }\end{aligned}$ & $\begin{array}{l}{ }^{1} \text { Turning point is well beyond } \\
\text { the range of observations }\end{array}$ \\
\hline 2004 & AMBIO & $\begin{array}{l}\text { 1. ODS** } \\
\text { 2. } \mathrm{CO} 2 \\
\text { 3. } \mathrm{CH} 4 \\
\text { 4. Total EF } \\
\text { 5. Forest EF } \\
\text { 6. Grazing EF } \\
\text { 7. Arable EF } \\
\text { 8. Fishing EF } \\
\text { 9. Built-up EF } \\
\end{array}$ & $\begin{array}{ll}\text { 1. } & 131 \\
\text { 2. } & 146 \\
\text { 3. } & 147 \\
\text { 4. } & 142 \\
\text { 5. } & 142 \\
\text { 6. } & 142 \\
\text { 7. } & 142 \\
\text { 8. } & 142 \\
\text { 9. } & 142 \\
\end{array}$ & $\begin{array}{ll}\text { 1. } & 1997 \\
\text { 2. } & 1996 \\
\text { 3. } 1991 \\
\text { 4. } 1996 \\
\text { 5. } 1996 \\
\text { 6. } 1996 \\
\text { 7. } 1996 \\
\text { 8. } 1996 \\
\text { 9. } 1996 \\
\end{array}$ & $\begin{array}{c}\text { >\$13K } \mathrm{K}^{1} \\
>\$ 34.8^{2} \\
\text { n.s. } \\
+ \text { Quadratic } \\
+ \text { Quadratic } \\
\text { n.s. } \\
\text { n.s. } \\
\text { n.s. } \\
\text { n.s. }\end{array}$ & $\begin{array}{l}{ }^{1} \text { Beyond the range of a vast } \\
\text { majority of nations } \\
{ }^{2} \text { Beyond the range of } \\
\text { observations }\end{array}$ \\
\hline 2004 & JIE & $\mathrm{EF} / \mathrm{GDP} / \mathrm{Md}^{\star \star \star \star}$ & 139 & $1998-9$ & N.A. & $\begin{array}{l}\text { Sensitivity analyses reveal the } \\
\text { need for extraordinary gain in } \\
\text { efficiency to counter footprint } \\
\text { effects }\end{array}$ \\
\hline 2005 & $\begin{array}{c}\text { Globalization \& The } \\
\text { Environment }\end{array}$ & $\begin{array}{l}\text { 1. } \mathrm{SO}_{2} \\
\text { 2. } \mathrm{NO}_{\mathrm{x}}\end{array}$ & $\begin{array}{ll}\text { 1. } & 138 \\
2 . & 138 \\
\end{array}$ & $\begin{array}{ll}\text { 1. } & 1995 \\
\text { 2. } & 1995 \\
\end{array}$ & $\begin{array}{l}>\$ 14.4 \mathrm{~K}^{1} \\
>\$ 23.3 \mathrm{~K}^{2}\end{array}$ & $\begin{array}{l}{ }^{1} 85 \% \text { of nations are below this } \\
{ }^{2} 99 \% \text { of nations are below this }\end{array}$ \\
\hline 2007 & $\begin{array}{l}\text { Frontiers in Ecology } \\
\text { and the Environment }\end{array}$ & $\begin{array}{l}\text { Total Ecological } \\
\text { Footprint (EF) }\end{array}$ & $128-135$ & 2001 & +Quadratic & \\
\hline
\end{tabular}

${ }^{1}$ Proceedings of the National Academy of Sciences

${ }^{2}$ Journal of Industrial Ecology

* GWP $=$ Global Warming Potential (A combination $\mathrm{CO}_{2}$ and $\mathrm{CH}_{4}$ )

** ODS = Ozone Depleting Substances (Chlorofluorocarbons (CFCs), halons, other fully halogenated CFCs, carbon tetracholoride, methyl, chloroform, HCFCs, and methyl bromide)

${ }^{\star \star *} \mathrm{Md}=$ The median of the EF/GDP Ratio 


\section{GENERAL FINDINGS:}

FOR EVERY IMPACT EXAMINED WE FIND:

- SUPPORT FOR CONTINUED IMPACT THEORIES (CURVE b)

- REJECTION OF NEO-LIBERAL /MODERNIZATION THEORIES OR THE KUZNETS CURVE (CURVE a) 


\section{SPECIFIC FINDINGS:}

FOR EVERY IMPACT EXAMINED:

- POPULATION IS ALWAYS A LEADING DRIVER OF IMPACTS

(1) CONSISTENTLY A PROPORTIONAL RELATIONSHIP (UNIT ELASTICITY)

- AFFLUENCE IS ALWAYS ALSO A LEADING DRIVER

(1) FOR $\mathrm{CO}_{2}$ THE RELATIONSHIP IS ALWAYS ELASTIC (COEFICIENT $\approx 1.5$ )

(2) FOR OTHER IMPACTS THE RELATIONSHIP IS INELASTIC (COEFICIENTS OF .26 TO .94)

- OTHER CONTRIBUTING FACTORS:

(1) POPULATION COMPOSITION, (2) LATTITUDE, AND (3) URBANIZATION 
ELEVEN INDEPENDENT AND CONTROL VARIABLES: ${ }^{*}=$ SMALL, SIGNIFICAN EFFECTS)

(1) *NON-DEPENDENT POPULATION (\% OF POPULATION BETWEEN 15 A 65)

(2) LAND AREA PER CAPITA

(3) *LATTITUDE (CLIMATIC ZONES)

(4) \% OF GDP IN NON-SERVICE SECTORS

(5) WHETHER SOCIETY IS CAPITALIST (VS. MIXED CAPITALIST OR CAPITALIST-STATIST)

(6) *\% URBAN POPULATION

(7) COUNTRY'S POSITION IN WORLD SYSTEM (CORE, SEMI-PERIPHERY, PERIPHERY)

(8) DIRECT FOREIGN INVESTMENT

(9) POLITICAL RIGHTS (FAIR AND OPEN ELECTIONS) 


\section{U.S. POpulation, \\ $\mathrm{CO}_{2}$ EMISSIONS, \& EnERgy CONSUIMTION Per Capita}

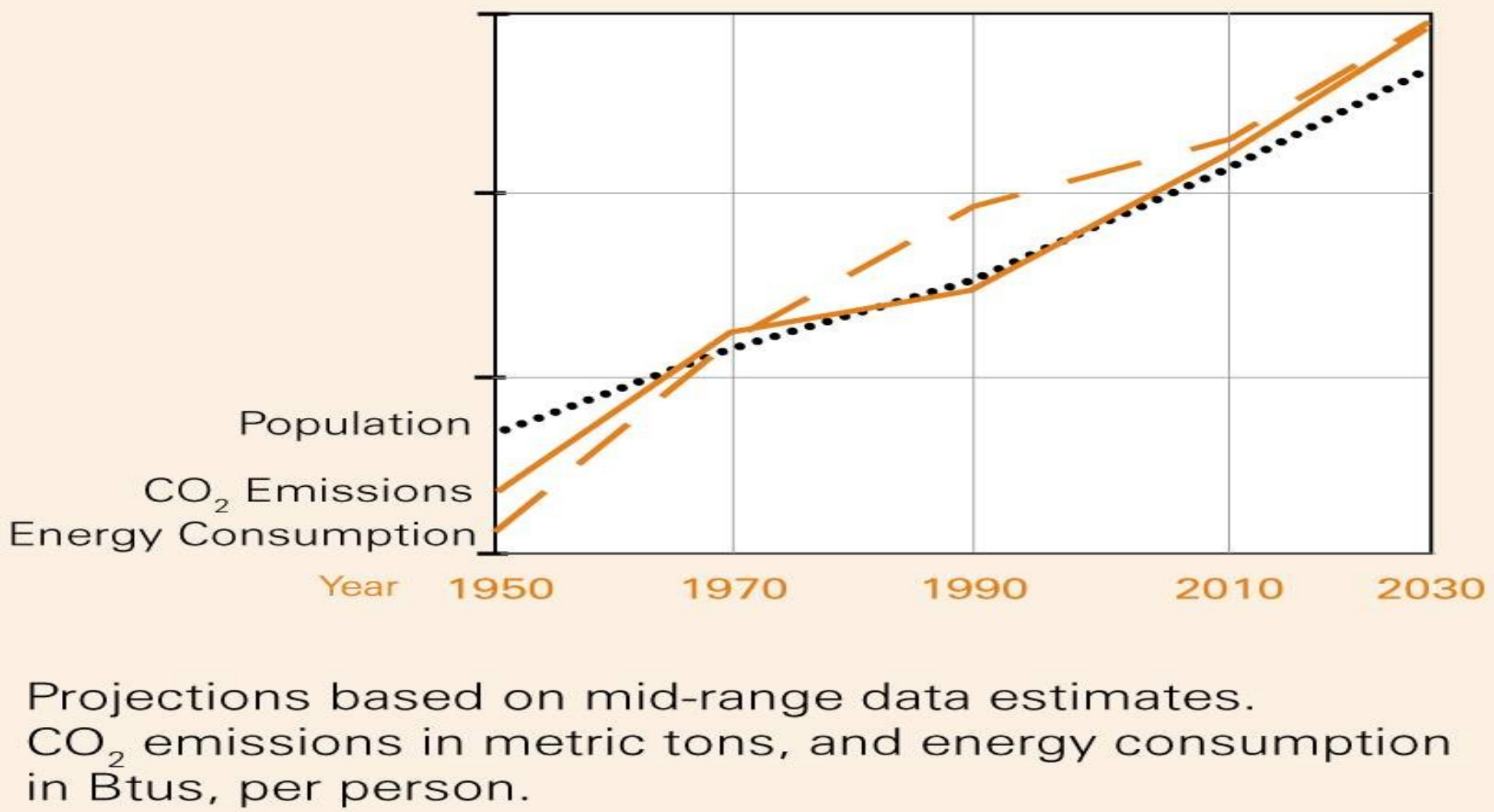

Source: Center for Environment and Population (CEP). WWW.cepnet.org, based on information from US Census Bureau, US EIA, WRI, 2008 
FOR ALL THESE STUDIES THE TECHNOLOGY FACTOR IS CONTAINED IN THE ERROR TERM OF THE STOCHASTIC EQUATION:

-THE ERROR TERM COMPRISES NOT ONLY TECHNOLOGY BUT ALL OTHER

VARIABLES (e.g. CULTURAL PRACTICES) NOT EXPLICITLY SPECIFIED IN THE

EQUATION.

-THIS EXAGGERATES THE ESTIMATED EFFECTS OF TECHNOLOGY.

WE HAVE ADDRESSED THIS ISSUE IN OUR WORK IN PROCESS:

-INDEX OF TRANSPORTATION INFRASTRUCTURE (KILOMETERS OF PAVED

ROADS, KILOMETERS OF RAIL LINES, AND ANNUAL AIR PASSANGERS

PER CAPITA) 
Longitudinal Analysis: Panel Analysis of Pooled Cross-Sections (Ecological Footprint EF)

\begin{tabular}{|c|c|c|c|c|c|c|c|c|c|c|c|c|}
\hline & Population & $\begin{array}{l}\text { Dep.Rati } \\
0\end{array}$ & GDPpc & $\mathrm{GDPpc}^{2}$ & $\%$ Urban & $\%$ Urban $^{2}$ & $\begin{array}{l}\text { Exports } \\
\% \text { GDP }\end{array}$ & $\begin{array}{l}\text { Imports } \\
\% \text { GDP }\end{array}$ & Const. & $\begin{array}{l}\text { Within } \\
\mathrm{R}^{2}\end{array}$ & $\begin{array}{l}\text { Number } \\
\text { Obsert. }\end{array}$ & $\begin{array}{l}\text { Number } \\
\text { Countr. }\end{array}$ \\
\hline Total EF & $\begin{array}{l}0.98^{*} \\
(0.19)\end{array}$ & $\begin{array}{l}-0.31^{*} \\
(0.07)\end{array}$ & $\begin{array}{l}-0.21^{*} \\
(0.08)\end{array}$ & $\begin{array}{l}0.03^{*} \\
(0.01)\end{array}$ & $\begin{array}{l}-0.72^{*} \\
(0.13)\end{array}$ & $\begin{array}{l}0.12^{*} \\
(0.02)\end{array}$ & $\begin{array}{l}-0.06^{*} \\
(0.01)\end{array}$ & $\begin{array}{l}0.07^{*} \\
(0.01)\end{array}$ & $\begin{array}{l}1.31^{*} \\
(0.44)\end{array}$ & .848 & 2247 & 65 \\
\hline $\begin{array}{l}\text { Energy } \\
\text { EF }\end{array}$ & $\begin{array}{l}0.99^{*} \\
(0.03)\end{array}$ & $\begin{array}{l}-0.48^{*} \\
(0.13)\end{array}$ & $\begin{array}{l}-0.28 \\
(0.16)\end{array}$ & $\begin{array}{l}0.05^{*} \\
(0.01)\end{array}$ & $\begin{array}{l}-1.60^{*} \\
(0.27)\end{array}$ & $\begin{array}{l}0.23^{*} \\
(0.04)\end{array}$ & $\begin{array}{l}-0.06^{*} \\
(0.02)\end{array}$ & $\begin{array}{l}0.09^{*} \\
(0.02)\end{array}$ & $\begin{array}{l}0.22 \\
(0.83)\end{array}$ & .824 & 2247 & 65 \\
\hline $\begin{array}{l}\text { Material }^{2} \\
\mathrm{EF}\end{array}$ & $\begin{array}{l}1.00^{*} \\
(0.19)\end{array}$ & $\begin{array}{l}-0.12^{*} \\
(0.05) \\
\end{array}$ & $\begin{array}{l}0.18^{*} \\
(0.15)\end{array}$ & & $\begin{array}{l}-0.33^{*} \\
(0.10) \\
\end{array}$ & $\begin{array}{l}0.08^{*} \\
(0.02)\end{array}$ & $\begin{array}{l}-0.05^{*} \\
(0.09) \\
\end{array}$ & $\begin{array}{l}0.05^{*} \\
(0.01)\end{array}$ & $\begin{array}{l}-0.92^{*} \\
(0.36)\end{array}$ & .813 & 2247 & 65 \\
\hline Food $\mathrm{EF}^{3}$ & $\begin{array}{l}0.99^{*} \\
(0.18)\end{array}$ & $\begin{array}{l}-0.12^{*} \\
(0.06)\end{array}$ & $\begin{array}{l}0.25^{*} \\
(0.07)\end{array}$ & $\begin{array}{l}-0.01^{*} \\
(0.00)\end{array}$ & $\begin{array}{l}-0.40^{*} \\
(0.11)\end{array}$ & $\begin{array}{l}0.10^{*} \\
(0.02)\end{array}$ & $\begin{array}{l}-0.05^{*} \\
(0.01)\end{array}$ & $\begin{array}{l}0.05^{*} \\
(0.01)\end{array}$ & $\begin{array}{l}-0.85^{*} \\
(0.39)\end{array}$ & .790 & 2247 & 65 \\
\hline Forest EF ${ }^{4}$ & $\begin{array}{l}1.10^{*} \\
(0.05)\end{array}$ & $\begin{array}{l}-0.11 \\
(0.16)\end{array}$ & $\begin{array}{l}-0.58^{*} \\
(0.21)\end{array}$ & $\begin{array}{l}0.06^{*} \\
(0.01)\end{array}$ & $\begin{array}{l}-0.18 \\
(0.10)\end{array}$ & & $\begin{array}{l}-0.08^{*} \\
(0.03)\end{array}$ & $\begin{array}{l}0.09^{*} \\
(0.03)\end{array}$ & $\begin{array}{l}-1.82 \\
(1.04)\end{array}$ & .348 & 2247 & 65 \\
\hline
\end{tabular}

${ }^{1}$ Amount to sequester $\mathrm{CO}_{2}+$ Fuelwood + Nuclear

${ }^{2}$ Total EF minus Energy EF +

${ }^{3}$ Cropland + Pasture Land + Fishing Area

${ }^{4}$ Timber + Fuelwood

Years: $1961-2003$

${ }^{*} \mathrm{P} \leq .05$ 


\section{Population of the "New Consumer" Countries (2006)}

China

India

Brazil

Russia

Mexico

Turkey

South Korea

Argentina

Malaysia
$1,328,474,000$

$1,151,751,000$

$189,323,000$

$143,221,000$

$105,342,000$

$73,922,000$

$48,050,000$

$39,134,000$

$26,114,000$

Total

$3,105,331,000$ 


\section{Scatterplot of Average Life Satisfaction Regressed on}

Ecological Footprint per capita.

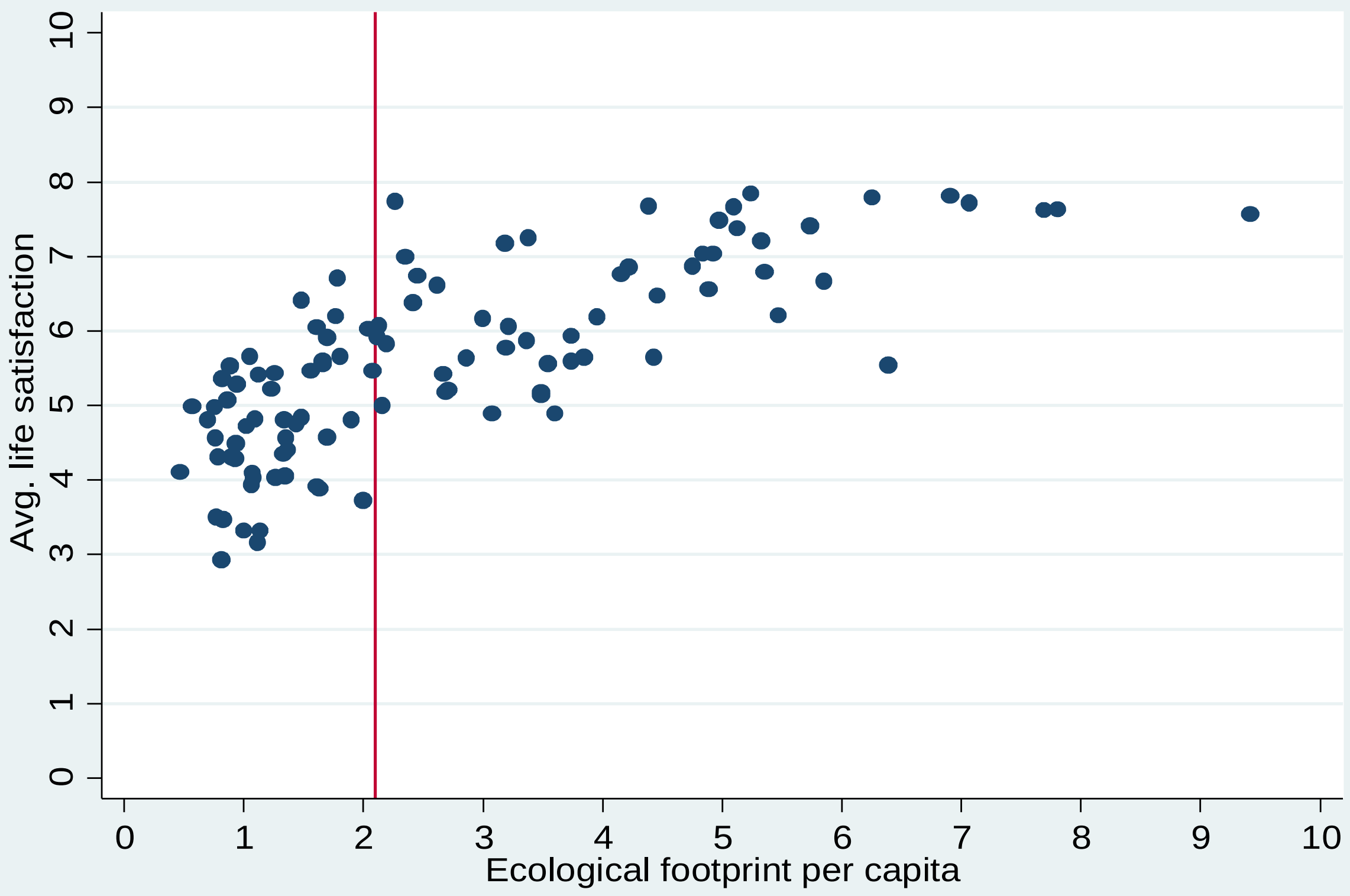




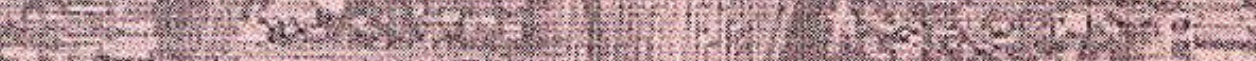

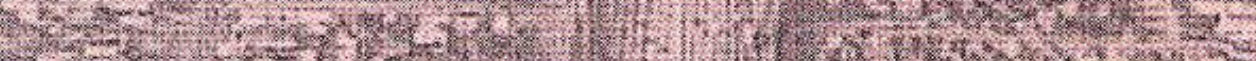

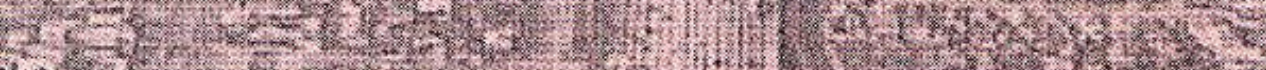

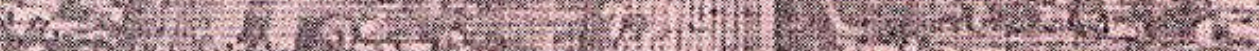

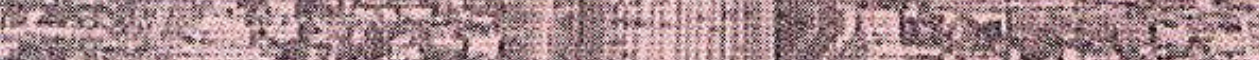

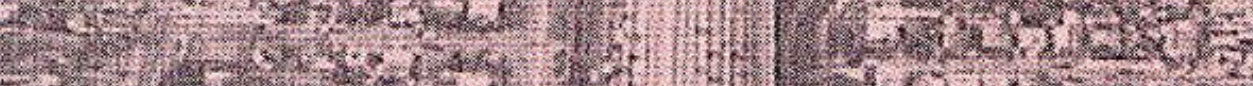

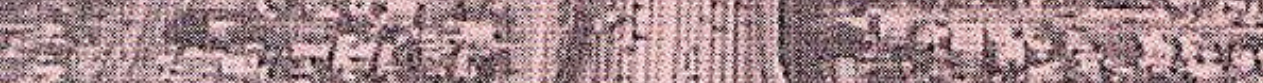

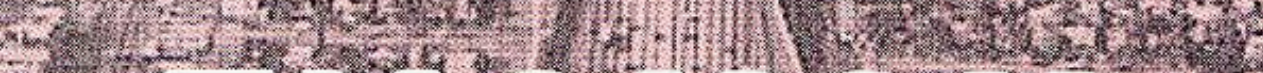

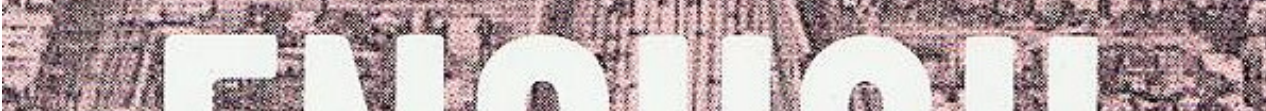

D

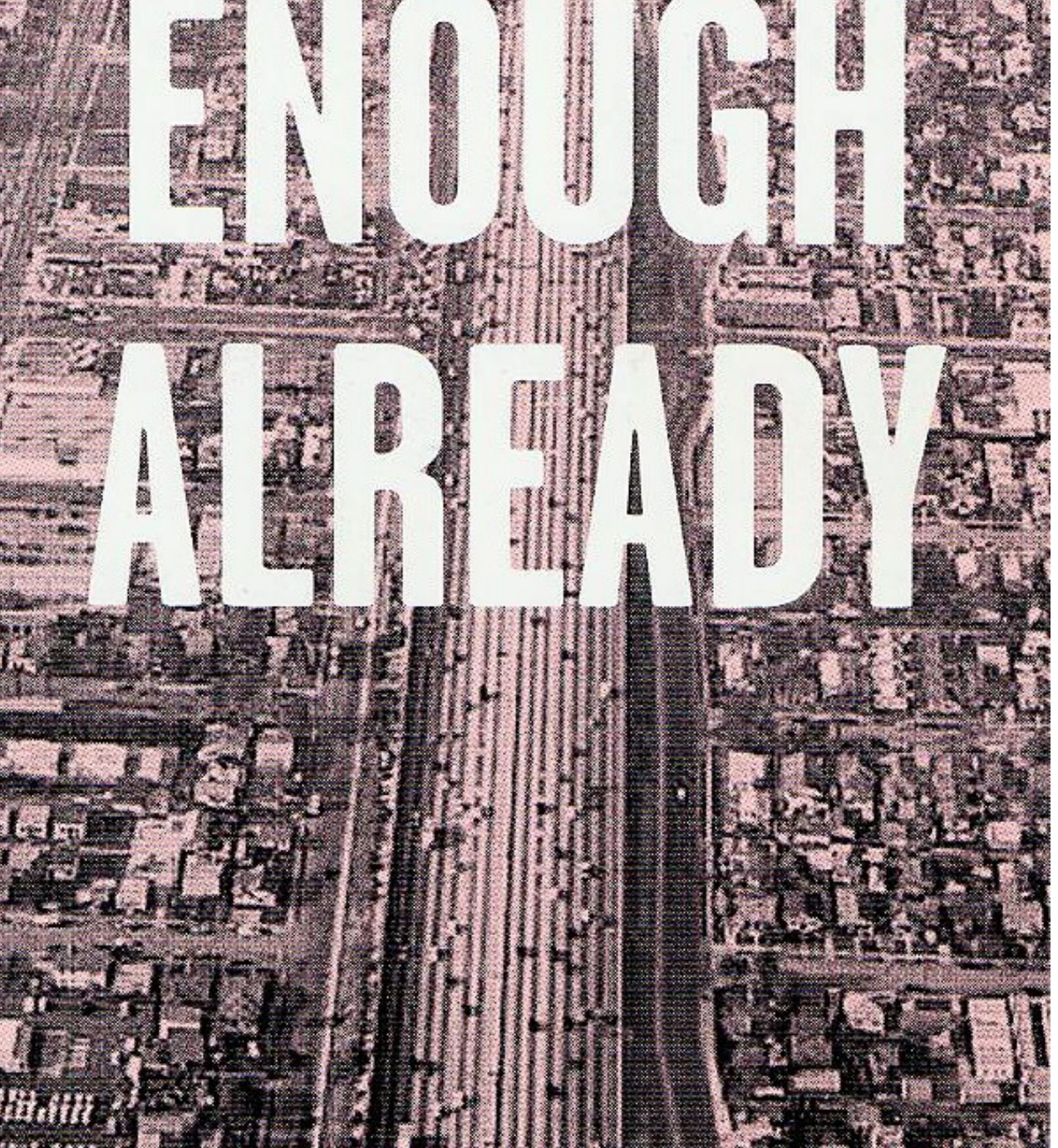




\section{THE LIMITS OF MODELS:}

IT IS IMPOSSIBLE TO SIMULTANEOUSLY MAXIMIZE GENERALITY, REALISM, AND PRECISION IN ANY MODEL.

THIS LIMITATION RESULTS IN THREE OPTIONS:

(1) SACRIFICE GENERALITY TO REALISM AND PRECISION

(2) SACRIFICE REALISM TO GENERALITY AND PRECISION

(3) SACRIFICE PRECISION TO REALISM AND GENERALITY

Source: Levins, Richard. 1966. "The Strategy of Model Building in Biology. American Scientist 54:421-431. 